\title{
Stress and sleep disturbance-a connection in CVD
}

Yuli Huang, Yunzhao Hu and Weiyi Mai

We read the Review by Steptoe and Kivimäki (Steptoe, A. \& Kivimäki, M. Stress and cardiovascular disease. Nat. Rev. Cardiol. 9, 360-370 [2012] $)^{1}$ with great interest. We wish to discuss some additional views about the important relationship between perceived stress, sleep disturbance, and cardiovascular disease (CVD).

Psychological stress is increasingly recognized as a potentially important risk factor for CVD. ${ }^{1}$ In the INTERHEART study, ${ }^{2}$ long-term exposure to stress was found to be an independent risk factor for acute myocardial infarction, even after taking into account combined exposure to conventional risk factors such as smoking, diabetes mellitus, hypertension, and obesity. However, the underlying mechanisms for the association between stress and cardiovascular risk have not been fully elucidated. Both direct physiological changes, such as elevations in epinephrine and norepinephrine levels, and indirect mechanisms, such as behaviors that increase CVD risk, have been suggested to be involved. ${ }^{1}$

Research has indicated that perceived stress correlates with disturbed sleep. ${ }^{3-5}$ Although sleep disturbance has not been identified as a specific risk factor in current cardiovascular prevention guidelines, an increasing number of studies have provided evidence that sleep disturbance is closely correlated with the incidence of CVD. ${ }^{6,7}$ In 2011, Laugsand and co-workers reported that insomnia was associated with a moderately increased risk of acute myocardial infarction during 11.4 years of followup in $>50,000$ individuals. ${ }^{6}$ Similarly, Hoevenaar-Blom and colleagues found an association between short sleep duration and the incidence of CVD in a prospective study of $>20,000$ adults (mean follow-up 12 years). ${ }^{7}$ These studies provide robust evidence supporting the pronounced impact of sleep disorders on the incidence of CVD. However, the correlation between perceived stress and sleep disorders was not explored in these studies.

In a paper published in June 2012, Kashani et al. reported that perceived stress correlates with disturbed sleep, as well as with other cardiovascular risk factors such as high BMI, increased waist circumference, and an elevated level of serum C-reactive protein (high-sensitivity assay). ${ }^{8}$ They concluded that the connection between stress and sleep might be an important mechanistic mediator of the association between stress and CVD. ${ }^{8}$ Bidulescu et al. found that sleep quality was associated with obesity in women, and was modified by perceived stress. ${ }^{9}$ Our research has demonstrated that perceived stress is associated with poor sleep quality and increased activation of the sympathetic nervous system, and that these factors are independent predictors of nondipping hypertension. ${ }^{10,11}$ We found that adding the mild sedative zolpidem to antihypertensive therapy improves sleep quality and stress status, decreasing sympathetic nervous system activity. ${ }^{12}$ In the Whitehall II study, ${ }^{13}$ work-related stress was associated with low levels of physical activity, poor diet, increased incidence of metabolic syndrome, and reduced heartrate variability, suggesting that stress has direct effects on the autonomic nervous system and neuroendocrine function. In addition, parasympathetic heart-rate variability has been reported to be decreased in healthy young adults during periods of perceived stress. ${ }^{14}$

Taken together, these observations suggest that perceived stress is associated with poor sleep quality and modulation of the autonomic nervous system. This mechanism could be the connection between stress and CVD. Prospective cohort studies are needed to assess the relationship between perceived stress, sleep disorders, and CVD, and to explore the underlying mechanisms, particularly variations in neuroendocrine function.
Department of Cardiology, the Affiliated Hospital at Shunde (the First People's Hospital of Shunde), Southern Medical University, Penglai Road 1, Daliang Town, Shunde District, Foshan 528300, People's Republic of China (Y. Huang, Y. Hu). Department of Cardiology, the First Affiliated Hospital of Sun Yat-sen University, 58 Zhongshan Road 2, Guangzhou 510080, People's Republic of China (W. Mai).

Correspondence to: W. Mai

wymai@hotmail.com

\section{Acknowledgments}

The authors are funded by the Medical Scientific Research Grant of the Health Ministry of Guangdong province, China (No: B2011310, No: A2012663),

Scientific Research Fund of Guangdong, China (No: 2009B3060700010, No: 2011B031800021), and Scientific Research Fund of Foshan, Guangdong, China (No: 2009,025 and 2011AA100473).

Competing interests

The authors declare no competing interests.

1. Steptoe, A. \& Kivimäki, M. Stress and cardiovascular disease. Nat. Rev. Cardiol. 9, 360-370 (2012).

2. Yusuf, S. et al. Effect of potentially modifiable risk factors associated with myocardial infarction in 52 countries (the INTERHEART study): case-control study. Lancet 364, 937-952 (2004).

3. Sing, C. Y. \& Wong, W. S. Prevalence of insomnia and its psychosocial correlates among college students in Hong Kong. J. Am. Coll. Health 59, 174-182 (2011).

4. Nomura, K., Nakao, M., Takeuchi, T. \& Yano, E. Associations of insomnia with job strain, control, and support among male Japanese workers. Sleep Med. 10, 626-629 (2009).

5. Hall, M. et al. Psychological stress is associated with heightened physiological arousal during NREM sleep in primary insomnia. Behav. Sleep Med. 5, 178-193 (2007).

6. Laugsand, L. E., Vatten, L. J., Platou, C. \& Janszky, I. Insomnia and the risk of acute myocardial infarction: a population study. Circulation 124, 2073-2208 (2011).

7. Hoevenaar-Blom, M. P., Spijkerman, A. M., Kromhout, D., van den Berg, J. F. \& Verschuren, W. M. Sleep duration and sleep quality in relation to 12-year cardiovascular disease incidence: the MORGEN study. Sleep 34, 1487-1492 (2011).

8. Kashani, M., Eliasson, A. \& Vernalis, M. Perceived stress correlates with disturbed sleep: a link connecting stress and cardiovascular disease. Stress 15, 45-51 (2012). 
9. Bidulescu, A. et al. Interaction of sleep quality and psychosocial stress on obesity in African Americans: the Cardiovascular Health Epidemiology Study (CHES). BMC Public Health 10, 581 (2010).

10. Huang, Y. et al. Poor sleep quality, stress status, and sympathetic nervous system activation in nondipping hypertension. Blood Press. Monit. 16, 117-123 (2011).

11. Huang, Y., Mai, W. \& Hu, Y. Relationship between sleep quality and the level and pattern of BP. Nat. Rev. Cardiol. 9, 429 (2012).

12. Huang, Y. et al. The effect of zolpidem on sleep quality, stress status, and nondipping hypertension. Sleep Med. 13, 263-268 (2012).
13. Chandola, T. et al. Work stress and coronary heart disease: what are the mechanisms? Eur. Heart J. 29, 640-648 (2008).

14. Matthews, S., Jelinek, H., Vafaeiafraz, S. \& McLachlan, C. S. Heart rate stability and decreased parasympathetic heart rate variability in healthy young adults during perceived stress. Int. J. Cardiol. 156, 337-338 (2012). 